University of Wollongong

Research Online

Faculty of Engineering and Information

Faculty of Engineering and Information

Sciences - Papers: Part B

Sciences

2016

Modeling and Identification of a Realistic Spiking Neural Network and

Musculoskeletal Model of the Human Arm, and an Application to the

Stretch Reflex

Manish Sreenivasa

University of Wollongong, manishs@uow.edu.au

Ko Ayusawa

National Institute of Advanced Industrial Science and Technology

Yoshihiko Nakamura

University of Tokyo

Follow this and additional works at: https://ro.uow.edu.au/eispapers1

Part of the Engineering Commons, and the Science and Technology Studies Commons

Research Online is the open access institutional repository for the University of Wollongong. For further information contact the UOW Library: research-pubs@uow.edu.au 


\title{
Modeling and Identification of a Realistic Spiking Neural Network and Musculoskeletal Model of the Human Arm, and an Application to the Stretch Reflex
}

\author{
Abstract \\ This study develops a multi-level neuromuscular model consisting of topological pools of spiking motor, \\ sensory and interneurons controlling a bi-muscular model of the human arm. The spiking output of motor \\ neuron pools were used to drive muscle actions and skeletal movement via neuromuscular junctions. \\ Feedback information from muscle spindles were relayed via monosynaptic excitatory and disynaptic \\ inhibitory connections, to simulate spinal afferent pathways. Subject-specific model parameters were \\ identified from human experiments by using inverse dynamics computations and optimization methods. \\ The identified neuromuscular model was used to simulate the biceps stretch reflex and the results were \\ compared to an independent dataset. The proposed model was able to track the recorded data and \\ produce dynamically consistent neural spiking patterns, muscle forces and movement kinematics under \\ varying conditions of external forces and co-contraction levels. This additional layer of detail in \\ neuromuscular models has important relevance to the research communities of rehabilitation and clinical \\ movement analysis by providing a mathematical approach to studying neuromuscular pathology.

\section{Keywords} \\ musculoskeletal, model, human, arm, application, stretch, reflex, identification, modeling, realistic, spiking, \\ neural, network \\ Disciplines \\ Engineering | Science and Technology Studies

\section{Publication Details} \\ Sreenivasa, M., Ayusawa, K. \& Nakamura, Y. (2016). Modeling and Identification of a Realistic Spiking \\ Neural Network and Musculoskeletal Model of the Human Arm, and an Application to the Stretch Reflex. \\ IEEE Transactions on Neural Systems and Rehabilitation Engineering, 24 (5), 591-602.
}




\title{
Modeling and Identification of a Realistic Spiking Neural Network and Musculoskeletal Model of the Human Arm, and an Application to the Stretch Reflex
}

\author{
Manish Sreenivasa, Member, IEEE, Ko Ayusawa, and Yoshihiko Nakamura, Fellow, IEEE
}

\begin{abstract}
This study develops a multi-level neuromuscular model consisting of topological pools of spiking motor, sensory and interneurons controlling a bi-muscular model of the human arm. The spiking output of motor neuron pools were used to drive muscle actions and skeletal movement via neuromuscular junctions. Feedback information from muscle spindles were relayed via monosynaptic excitatory and disynaptic inhibitory connections, to simulate spinal afferent pathways. Subject-specific model parameters were identified from human experiments by using inverse dynamics computations and optimization methods. The identified neuromuscular model was used to simulate the biceps stretch reflex and the results were compared to an independent dataset. The proposed model was able to track the recorded data and produce dynamically consistent neural spiking patterns, muscle forces and movement kinematics under varying conditions of external forces and co-contraction levels. This additional layer of detail in neuromuscular models has important relevance to the research communities of rehabilitation and clinical movement analysis by providing a mathematical approach to studying neuromuscular pathology.
\end{abstract}

Index Terms-Biological systems modeling, neural engineering, neurophysiological parameter identification, stretch reflex.

\section{SymbOLS AND DESCRIPTIONS}

*subscripts $i, j$ indicate Biceps (B) or Triceps $(T)$

$\begin{array}{ll}q & \text { Joint angle (radians). } \\ r & \text { Joint torque (Nm). } \\ f & \text { Muscle force }(\mathrm{N}) . \\ a & \text { Muscle activation. }\end{array}$

Manuscript received September 22, 2014; revised May 12, 2015; accepted September 07, 2015. Date of publication September 17, 2015; date of current version May 06, 2016. This work was supported by the HPCI STRATEGIC PROGRAM Computational Life Science and Application in Drug Discovery and Medical Development by MEXT, Japan, and, the Alexander von Humboldt Foundation, Germany

A preliminary version of this study appeared in the proceedings of the 2013 IEEE International Conference on Intelligent Robots and Systems (IROS).

M. Sreenivasa is with the Optimization in Robotics and Biomechanics Group, Interdisciplinary Center for Scientific Computing, University of Heidelberg, 69115 Heidelberg, Germany (e-mail: manish.sreenivasa@iwr.uni-heidelberg. de).

K. Ayusawa is with the CNRS-AIST Joint-Robotics Laboratory, Intelligent Systems Research Institute, National Institute of Advanced Industrial Science and Technology (AIST), 305-8560 Tsukuba, Japan.

Y. Nakamura is with the Department of Mechanoinformatics, University of Tokyo, 113-8656 Tokyo, Japan.

Color versions of one or more of the figures in this paper are available online at http://ieeexplore.ieee.org.

Digital Object Identifier 10.1109/TNSRE.2015.2478858

$\begin{array}{ll}v & \text { Muscle velocity (m/s). } \\ l_{N} & \text { Muscle residual length (m). } \\ V & \text { Neural membrane voltage }(\mathrm{V}) . \\ C & \text { Neural membrane capacitance }(\mathrm{F}) . \\ \tau & \text { Neural time constant (s). } \\ I_{\mathrm{syn}} & \text { Synaptic current (A). } \\ u & \text { Spike events. } \\ \psi & \text { Spindle firing frequency (Hz). } \\ S_{j} & \text { Stimulation (A). } \\ D_{S F, j} & \text { Soma diameter scale factor. } \\ D_{i, j} & \text { Soma diameter (m). } \\ F_{S F, j} & \text { Motor unit maximum force scale factor. } \\ F_{i, j} & \text { Motor unit maximum force (N). } \\ T_{S F, j} & \text { Motor unit time to maximum force scale factor. } \\ T_{i, j} & \text { Motor unit time to maximum force (s). } \\ r_{S F} & \text { Resistive torque scale factor. } \\ \omega_{i j \pm} & \text { Pool } i \rightarrow j \text { synaptic center weight. } \\ \sigma_{i j \pm} & \text { Pool } i \rightarrow j \text { synaptic variance. } \\ & \end{array}$

\section{INTRODUCTION}

$\mathbf{M}$ ODELING of human neuromuscular physiology plays an important role in furthering the state-of-the-art technology in rehabilitation. In addition to extending our knowledge of human motor control, such models find applications in the design and control of human-machine interfaces such as neuro-prostheses and powered exoskeletons, as well as in the detection of neuromuscular pathology [1]-[6]. In recent research, neuromuscular models have been applied to the simulation of a wide variety of movements. These studies share methods such as multi-body kinematics and dynamics, optimization and mathematical modeling, with robotics research. It is notable that often the neural component is simplified as a one-dimensional scalar per muscle signifying the activation level, $a$, ranging from 0 to 1 . This is a gross approximation as individual muscles may be driven, not by one-dimensional inputs, but several hundreds to thousands of simultaneous spike 
trains from spinal motorneurons. Nevertheless, within reasonable constraints, neuromuscular models of this type have shown promise in estimating and predicting a wide range of macroscopic movements such as disturbance compensation, locomotion and the stretch reflex [3], [7]. The simplified models have been used to study and explain the conceptual mechanism of the neuromuscular system. However, as a limitation of the simplified neural architecture, more detailed aspects of neuromuscular control such as the recruitment principle and rate coding [8] are difficult to capture.

The more complex level of modeling involves decomposing the neural components of muscle control by incorporating neuronal morphology. This extends the scope of the investigation from qualitative concepts of neuromuscular action to quantitative processes. There are relatively few studies that simultaneously investigate these aspects of spinal neural and musculoskeletal architecture (see, for example, [4]-[6], [9]-[13]). At the simplest level, neural command of muscle action is the flow of information (spike trains) from spinal $\alpha$-motor neurons to the innervated muscle fibres. However, this is further qualified by interacting and interconnected pools of the spinal cord interneurons, proprioceptive feedback information from muscle spindles/Golgi-tendon organs, and modulation from higher neural centers all the way to the motor cortex of the brain. It is vital for any model to capture this closed loop nature of the neuromuscular system, as clinically observed pathologies may only be fully explained in the context of feedback dynamics [2], [5], [6]. Note that here we refer not only to the feedback from the skeletal muscles, but also the intra-spinal loops that serve to coordinate and modulate reflexes as well as other complex behaviors.

There are various challenges involved in reproducing this entire circuitry in a mathematical model. For example, much of the inner architecture is still unknown and it is not yet possible to record neural signals in vivo, making it difficult to validate such models. Additionally, it is computationally infeasible to simulate the behavior of billions of individual neurons simultaneously. Studies thus concentrate on subsets of neural networks specific to tasks such as reflexes [4]-[6], [9], [10], or spinal motor control under isometric conditions [11]. To relate to the additional neural detail, the corresponding muscle actuation also needs to be more complex. For example, motor-unit level force generation models [11], [14] or collection of Hill-type constructs [12] approximate the macroscopic muscle characteristics, whereas detailed continuum mechanics models simulate the molecular level dynamics [13]. Based on these perspectives, neuromuscular model choices may be considered as a trade-off between 1) approximating complex neuromuscular behavior by mathematical functions that are reasonably simple, and 2) determining parameters for these functions that can accurately reproduce behavior beyond those recorded.

This study bridges the gap between physiologically realistic topological neural networks and rigid-body musculoskeletal systems, by modeling and identifying the parameters of a combined NMS model of the arm. In contrast to similar studies on spiking neuromuscular models, here we base our models on physiologically realistic scales and ranges identified from experimental data. The scenario tested is the stretch reflex of

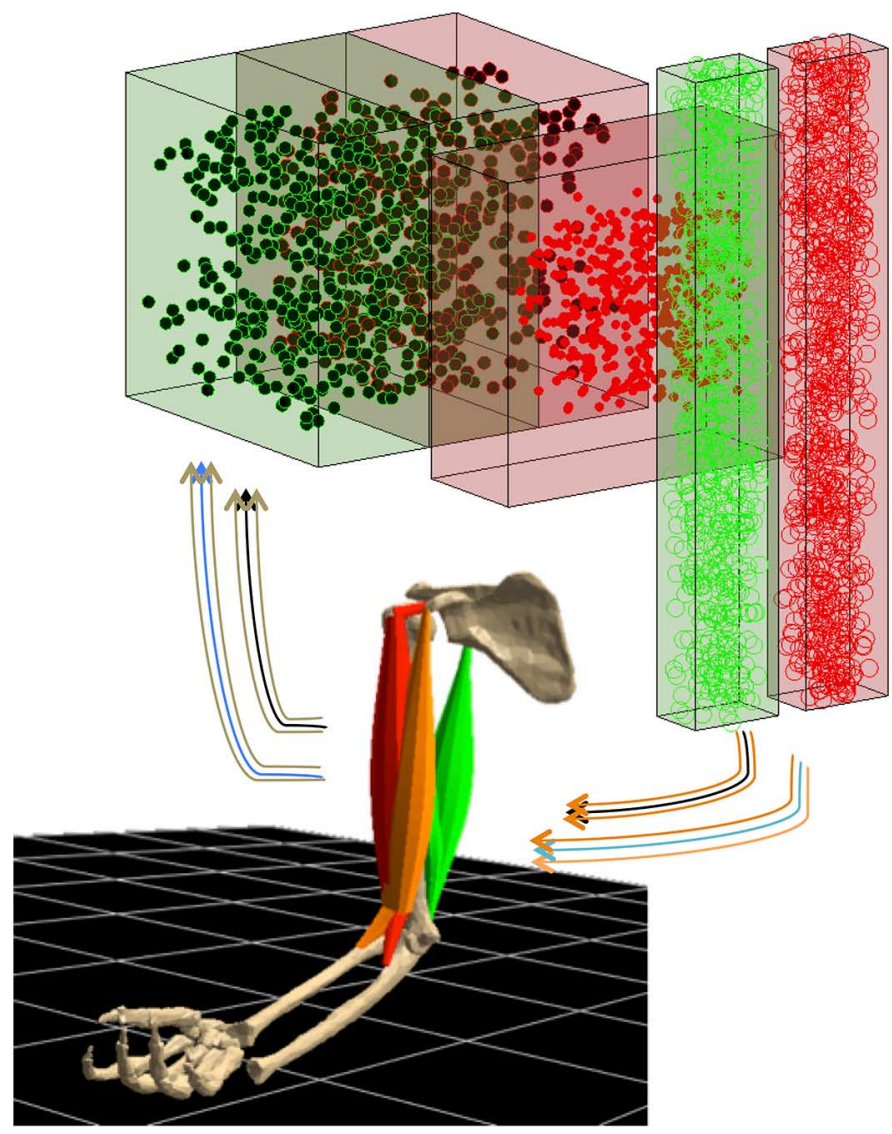

Fig. 1. NMS model with a topological neural network: In the arm posture shown above, a stretch in the biceps muscle spindles causes the corresponding excitation of the biceps motor neurons, and inhibition of the triceps motor neurons. Neurons were randomly distributed inside fixed volumes to simulate neural pools in the spine.

the biceps muscle in the posture illustrated in Fig. 1. Here, we use a simple model of the arm mechanics (1 DoF, two wire-muscles), and limit our analysis to the $I a$ reflex loop. The purpose of this simplification is to focus this study on the model identification methods at the motor unit level, while still incorporating the effects of skeletal movements and feedback. In the following sections, we detail our neuromuscular model, the human experimental paradigms and the parameter identification methodology.

\section{Neuromusculoskeletal Model}

The network architecture and neural dynamics detailed in this section were implemented in the software NEST [15]. Musculoskeletal forward kinematics and dynamics were simulated using a custom-built software using robotics algorithms and musculoskeletal visualization, sDIMS [16]. Communication between the NEST and sDIMS simulators was implemented in the Message Parsing Interface (MPI) parallel computing environment using the MUSIC framework [17]. Fig. 2 illustrates the overall system dynamics.

\section{A. Neural Network}

Fig. 1 shows an overview of the NMS model developed in this study. The neural network consisted of topological distributions of Motor Neurons (MN), Sensory Neurons (SN) and Inter 


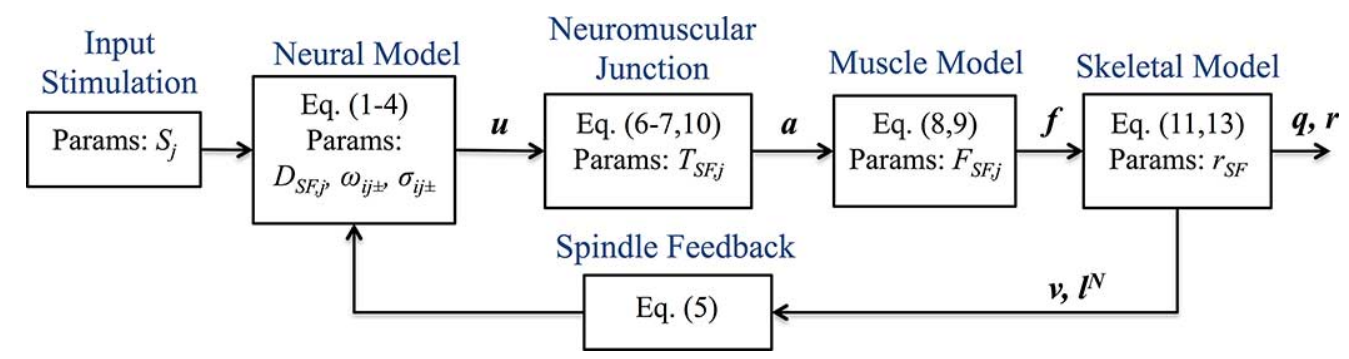

Fig. 2. Schematic of model dynamics: Refer to Section I for description of parameters. Subscript $j$ refers to the biceps/triceps.

Neurons (IN). Stimulations, $S_{B}$ and $S_{T}$, were applied as direct currents to the $\mathrm{MN}$ pools of the biceps and triceps, respectively. Within each pool stimulation was applied to all motor neurons uniformly. These stimulations represented the cumulative descending outputs from upper spinal neural pools and the brain.

1) Alpha Motor Neurons: For our two muscle system we chose the number of Motor Units (MUs) based on counts from human physiology [18], [19] as, $N_{M U, B}=774$ for the biceps, and $N_{M U, T}=717$ for the triceps. Note that the triceps MU number was not directly available and was approximated from muscle spindle to motor axon ratio [20]. Dynamic behavior of MNs was modeled by the computationally efficient leaky Integrate And Fire (LIF) neuron model [21]. The LIF model represents the electrical behavior of one neuron as

$$
\frac{d V}{d t}=-\left(\frac{V-E}{\tau}\right)+\frac{I_{\mathrm{syn}}(t)}{C}+\frac{I_{e}}{C}
$$

where $V$ is the membrane potential, $E$ the resting membrane potential, $\tau$ the membrane time constant, $I_{\text {syn }}(t)$ the sum of alpha-shaped synaptic currents, $I_{e}$ the external input current and $C$ the membrane capacitance. The incoming currents, $I_{\text {syn }}(t)$ from other pre-synaptic neurons or $I_{e}$ from an external source, cause a build up of the neuron membrane potential. For persistent input currents, the membrane potential $V$ reaches a pre-defined threshold and fires a spike causing a rapid discharge of the potential and a reset to its resting value $E$. In the LIF model used here, the dynamics of this mechanism are primarily governed by the terms $\tau$ and $C$.

Motor neurons innervating a muscle have varying dynamic properties. MNs with a smaller soma surface area have a larger input resistance and for a given synaptic input reach their firing threshold earlier than bigger MNs. This arrangement of MNs by size results in an orderly recruitment based on the magnitude of synaptic inputs and is known as the recruitment principle [18], [22]. For the LIF neurons modeled in this network we mimic this variation of electrical properties as a function of $\mathrm{MN}$ soma diameter. Cat spinal MN diameters have been found to vary from $77.5 \mu \mathrm{m}$ to $113 \mu \mathrm{m}$ [11], [23]. Allowing for a larger variation in human MN diameters from $40.0 \mu \mathrm{m}$ to $140.0 \mu \mathrm{m}$, we modeled its distribution as a logarithmically increasing function of $\mathrm{MN}$ number ( $1=$ smallest, $\mathrm{N}=$ largest $)$

$$
D_{i, j}=\left[d_{m x}-d_{m n} \log \left(N_{j}-i\right)\right] D_{S F, j}
$$

where, $j$ refers to the Biceps(B) or Triceps(T) and $D_{i, j}$ the soma diameter of the $i_{t h} \mathrm{MN}, d_{m x}$ and $d_{m n}$ are constants defined in Table I that determine the range of the distribution, $N_{j}$ is the total number of MNs in that neural pool, and $D_{S F, j}$ an adjustable scaling factor used in the parameter identification stage. Membrane capacitance, $C$ and the time constant, $\tau$ were computed for each $\mathrm{MN}$ based on this distribution of soma diameters, using

$$
\begin{aligned}
C_{i, j} & =\pi D_{i, j}^{2} c_{\mathrm{spf}} \\
\tau_{i, j} & =\tau_{\max }-\left(D_{i, j}-\tau_{a d j}\right) * \tau_{s l p} .
\end{aligned}
$$

The absolute ranges for these parameters were based on the values reported in [23], and adjusted to allow for a larger variation in human spinal MNs. $\tau_{i, j}$ was allowed to range from 14.38 $\mathrm{ms}$ to $3.05 \mathrm{~ms}$ according to (4), and proportionally mapped to the soma diameter, i.e., the smallest neuron had the highest time constant which linearly decreased with increasing neuron size. $C_{i, j}$ was calculated assuming a spherical surface area of the soma and a specific capacitance $c_{\mathrm{spf}}=1 e 4 \mu \mathrm{F} / \mathrm{m}^{2}$ [23]. Note that these ranges defined the maximum and minimum limits for the model. Their exact values within these ranges were determined in the parameter identification stage based on the scaling factors. In addition, we set a constant refractory period of $2 \mathrm{~ms}$ limiting the maximum discharge rate to $500 \mathrm{~Hz}$ [21]. The constants that determined the distributions in (2)-(4) are defined in Table I. Further details of the neuron model and integration scheme for the sub-threshold dynamics can be found in [21].

2) Ia Sensory Afferents: In the present study we only model the reflex dynamics due to the $I a$ primary afferent response of muscle spindles, that carry information about muscle length and velocity [24]. Each muscle spindle (MS) in the muscle body is associated with one SN located just outside the central spinal pools. In a comparison of various spindle models, Prochazka \& Grassini [25] commented on the efficiency of simple power-law models in representing the macroscopic firing characteristics of cat muscle spindles. The complete power law model included contributions from muscle velocity, muscle length, and a constant component representing the background firing of the spindle. Here, we modified the power law model to encode the Ia afferent response

$$
\psi(t)=4.3 v(t)^{0.6}+2 l_{N}(t)+10 .
$$

Frequency of spindle spikes $\psi(t)$ was calculated from muscle velocities $v(t)$ and residual length $l_{N}(t)$ in the forward dynamics simulation. Residual length, $l_{N}$, was calculated as the difference between the current and resting length of the muscle. We added a constant component of $10 \mathrm{~Hz}$ to the spindle firing frequency. In the original study [25], the authors used a higher frequency of $82 \mathrm{~Hz}$ to account for the background firing in the 
TABLE I

NMS IDENTIFIED MODEL

\begin{tabular}{|c|c|c|c|c|}
\hline \multicolumn{5}{|l|}{ Neural Components } \\
\hline & \# Components & Fixed Parameters & Identification Range & Model Identified Ranges \\
\hline Biceps MN & $N_{M U, B}=774$ & \multirow{2}{*}{$\begin{array}{c}E=-70 m V \\
d_{m n}=120.0, d_{m x}=8.5 \\
c_{s p f}=1 e 4 \mu F / m^{2} \\
\tau_{m a x}=12.5, \tau_{a d j}=60.0 \mu \\
\tau_{s l p}=9.44 e-2\end{array}$} & \multirow[b]{2}{*}{$40 \mu m \leq D \leq 140 \mu m$} & $\begin{array}{c}\mathbf{5 7 . 0 8 \mu m} \leq \boldsymbol{D}_{B} \leq \mathbf{1 0 9 . 3 7 \mu m} \\
102.32 p F \leq C_{B} \leq 375.62 p F \\
7.83 m s \leq \tau \leq 12.77 m s\end{array}$ \\
\hline Triceps MN & $N_{M U, T}=717$ & & & $\begin{array}{c}\mathbf{5 0 . 8 3 \mu \boldsymbol { m }} \leq \boldsymbol{D}_{\boldsymbol{T}} \leq \mathbf{1 0 3 . 9 9} \boldsymbol{\mu m} \\
81.14 p F \leq C_{T} \leq 339.59 p F \\
8.34 \mathrm{~ms} \leq \tau \leq 13.36 \mathrm{~ms}\end{array}$ \\
\hline Interneurons & $N_{I, B}=320$ & $\begin{array}{c}E=-70 \mathrm{mV} \\
\tau=10 \mathrm{~ms}, C=160 p F\end{array}$ & - & ( \\
\hline $\begin{array}{l}\text { Biceps MS } \\
\text { to Biceps MN }\end{array}$ & $N_{M S, B}=320$ & \multirow{2}{*}{ Eq. (5) } & \multirow{3}{*}{$\begin{array}{l}0 \leq w \leq 1 \\
0 \leq \sigma \leq 1\end{array}$} & $\begin{array}{c}\text { normalized [min,max] } \\
\boldsymbol{w}_{B B}+=[0.64,0.86] \\
\sigma_{B B}+=[0.55,0.77]\end{array}$ \\
\hline Triceps MS & $N_{M S, T}=520$ & & & $\begin{array}{c}\boldsymbol{w}_{T T+}=[0.4,0.69] \\
\sigma_{T T+}=[0.35,0.49]\end{array}$ \\
\hline $\begin{array}{l}\text { Biceps MS } \\
\text { to Interneurons }\end{array}$ & - & - & & $\begin{array}{l}w_{B I+}=[0.44,0.46] \\
\sigma_{B I+}=[0.36,0.41]\end{array}$ \\
\hline $\begin{array}{l}\text { Interneurons } \\
\text { to Triceps MN }\end{array}$ & - & - & $\begin{aligned}-1 & \leq w_{I T-} \leq 0 \\
0 & \leq \sigma_{I T-} \leq 1\end{aligned}$ & $\begin{array}{c}w_{I T-}=[-0.44,-0.45] \\
\sigma_{I T-}=[0.35,0.41]\end{array}$ \\
\hline \multicolumn{5}{|c|}{ Musculoskeletal Components } \\
\hline $\begin{array}{c}\text { Biceps MU } \\
\text { Maximum Force }\end{array}$ & $N_{M U, B}=774$ & \multirow{2}{*}{$\begin{array}{c}p_{m x}=15.0 \\
p_{m n}=2.25 \\
\text { Eq. (9) }\end{array}$} & \multirow{2}{*}{$0.03 N \leq F \leq 22.0 N$} & $0.0165 N \leq F_{B} \leq 18.19 N$ \\
\hline $\begin{array}{c}\text { Triceps MU } \\
\text { Maximum Force }\end{array}$ & $N_{M U, T}=717$ & & & $0.0124 N \leq F_{T} \leq 20.2 N$ \\
\hline $\begin{array}{c}\text { Biceps MU } \\
\text { Time to Max Force }\end{array}$ & $N_{M U, B}=774$ & \multirow{2}{*}{$\begin{array}{c}s_{m n}=0.125 \\
s_{s l}=0.245 \\
\text { Eq. }(10)\end{array}$} & \multirow{2}{*}{$5 m s \leq T \leq 250 m s$} & $32.2 m s \leq T_{B} \leq 175.0 \mathrm{~ms}$ \\
\hline $\begin{array}{c}\text { Triceps MU } \\
\text { Time to Max Force }\end{array}$ & $N_{M U, B}=717$ & & & $28.2 \mathrm{~ms} \leq T_{T} \leq 179.0 \mathrm{~ms}$ \\
\hline Resistive torque & - & Eq. 11 & $0.0 \leq r_{S F} \leq 3.0$ & $r_{S F}=[0.577,0.756]$ \\
\hline
\end{tabular}

cat biceps Femoris muscle. We opted for a lower background frequency based on studies on human spindle firing in the forearm and hand muscles [26]. The discrete spike events corresponding to the spindle frequency were approximated using a Poisson process [15]. Spindles were uniformly distributed in the corresponding muscle heads. The number of Ia afferents were based on allometric counts with $N_{M S, B}=320$, in the biceps muscle and, $N_{M S, T}=520$, in the triceps muscle [19].

3) Ia Interneurons: Connections between the sensory and motor neuron pools can be mono-synaptic or di-/poly-synaptic via intermediary pools made up of interneurons, Renshaw cells, etc. Here, we model one interneuron pool, the Ia Interneurons, to simulate the di-synaptic antagonist inhibitory action that is vital to the human stretch reflex. The role of this pathway is to detect the firing of the agonist (biceps) sensory neurons, and inhibit the action of the antagonist (triceps) motor neurons. We modeled these neurons as identical low capacitance LIF neurons with the default parameters listed in Table I. Spinal interneurons specific to a certain function, for example the Ia inhibition, are not easily identifiable in the human spine. We assumed a 1:1 ratio between the Ia Interneurons and the biceps sensory neurons (i.e., $N_{I, B}=N_{M S, B}=320$ ), to ensure good coverage between the pre-synaptic neurons and their targets. Note that here we only activate the IN pool responsible for inhibition of the triceps muscle.

4) Topology and Synaptic Connections: The neurons were organized topologically to approximate their arrangement in the human spine, Fig. 1. The neural layer consisted of 5 pools: $2 \mathrm{MN}$ pools, $2 \mathrm{SN}$ pools, and $1 \mathrm{IN}$ pool. The sizes and relative distances between the pools were chosen such that they approximated their arrangement in the human spinal column [22]. $\mathrm{MN}$ pools for each muscle were arranged in vertical ventral columns. The positions of the MNs were randomly distributed inside these columns. SN pools were located dorsally with neurons randomly distributed in a cuboidal volumn. IN pools were located between the $\mathrm{MN}$ and $\mathrm{SN}$ pools. The synaptic strengths were determined by this topological arrangement and the distance between a pre-synaptic neuron and its target [27]. Two types of pathways connected the various neuron pools; Monosynaptic Excitation (positive feedback to agonist $\mathrm{MN}$ pool), and, Disynaptic Inhibition (negative feedback to antagonist $\mathrm{MN}$ pool).

Synaptic characteristics between source and target neurons were modeled such that closer cells formed stronger synapses [11], [27]. Distant-dependent synaptic weight was varied using a symmetrical Gaussian distribution with center weight $\omega$ and variance $\sigma$. As the synaptic weight dropped quickly for larger distances, this implied that target neurons very far from the source were not influenced by it. Although this was potentially possible for very large values of $\sigma$ (see further comments in Section IV-D). Thus, for our network all synaptic values could be determined from the following parameters.

- $\omega_{B B+} \& \sigma_{B B+}$ : Maximum weight and variance of synaptic distribution for biceps $\mathrm{SN}$ to biceps $\mathrm{MN}$ connections, the ' + ' denotes excitation.

- $\omega_{T T+} \& \sigma_{T T+}$ : Triceps SN to triceps MN.

- $\omega_{B I+} \& \sigma_{B I+}$ : Biceps SN to IN

- $\omega_{I T-} \& \sigma_{I T-}:$ IN to triceps MN, the "_," denotes inhibition.

Delays due to axonal conduction time were fixed at $5 \mathrm{~ms}$ from $\mathrm{MN}$ to muscle fibres and from muscle spindles to sensory neurons (approximated using an axon length of $0.4 \mathrm{~m}$ and average conductance speed of $70 \mathrm{~m} / \mathrm{s}$ [8], [11], [28]), and $2 \mathrm{~ms}$ for all intra-spinal connections [29]. 


\section{B. Neuromuscular Junction}

The output of the neural network consisted of $N_{M U, B}+$ $N_{M U, T}$ efferent channels containing alpha $\mathrm{MN}$ spike events. These spikes result in MU twitch forces via electro-chemical processes occurring at the motor end plates, the junction of the $\mathrm{MN}$ axons and the muscle fibres. We decomposed this process into two parts: 1) neural spike to activation 2) activation to MU force. The latter part is detailed in Section II-C. Neural spike to activation was modeled as the impulse response of a second-order system [14]. We used a modification of the discrete time equations developed by Cisi and Kohn [11]

$$
\begin{aligned}
a(t)= & 2 \exp \left(\frac{-t}{T}\right) a(t-1)-\exp \left(\frac{-2 t}{T}\right) a(t-2) \\
& +\frac{t^{2}}{T} \exp \left(1-\frac{t}{T}\right) u(t)
\end{aligned}
$$

with, $u(t)= \begin{cases}1, & \text { if spike detected } \\ 0, & \text { if no spike detected }\end{cases}$

where, $a(t)$ was the activation at time instance $t$ and $T$ the time to maximum force. Note that (6) gives us the instantaneous activation level of one MU associated with the spiking behavior of one MN. $T$ was a distribution over each muscle's MUs and is explained further next.

\section{Musculoskeletal Model}

The arm was modeled as a fixed upper arm connected to the forearm by a rotational elbow joint, Fig. 1. The skeletal bones were scaled linearly to the subject-specific dimensions and corresponding mass and inertia properties approximated from anthropometric data [16]. The elbow joint was actuated by means of massless wires representing the biceps and triceps muscles. The biceps muscle consisted of two heads-biceps brachii caput breve and biceps brachii caput longum-arising from the scapula, and attaching to the radius and ulna bones of the forearm. The triceps muscle consisted of three heads - triceps brachii caput laterale, triceps brachii caput mediale and triceps brachii caput longum - arising from scapula and humerus, and attaching to the posterior olecranon process of the ulna. Muscle origins, insertion points and muscle lines-of-action were defined using via-points identified from physiology.

MU activations $a(t)(6)$, were used to calculate MU twitch forces at each time step. Activation to twitch force was computed using the Hill-type muscle model equation (see [30] for an overview)

$$
f(t)=a(t) f_{l}(l) f_{v}(v) F
$$

which relates muscle force $f(t)$ to the instantaneous activation level $a(t)$ and maximum force $F$. The terms $f_{l}(l)$ and $f_{v}(v)$ are normalized coefficients that are dependent on the muscle length and muscle velocity, and were computed based on muscle kinematics at each time step. Equation (8) approximates the force generated by the contractile element of the muscle. To simplify the muscle model we did not include the tendon dynamics or the fibre pennation angle. The summation of all MU twitches at one time step gave the total muscle force.

Similar to motor neurons, muscle fibres also have varying dynamic characteristics. In literature, muscle fibre bundles are often classified as S (Slow), FF (Fast Fatigue), or FR (Fatigue Resistant). This classification is based on a variation of maximum force $(F)$ and the time to maximum force $(T)$ of the muscle fibre bundles. Studies on cat motor units have shown that these distributions are more likely a continuous variation of $F$ and $T$ values rather than three distinct groups [28]. However, we acknowledge that recent studies on classifying the thenar muscle of the hand have questioned whether a similar association exists for human motor units [31]. Here, we mimic the relation originally reported by Burke et al. [28] by the distributions

$$
\begin{aligned}
& F_{i, j}=\left[p_{m x}-p_{m n} \log \left(N_{j}-i\right)\right] F_{S F, j} \\
& T_{i, j}=\left[s_{m n}-\frac{s_{s l}}{N_{j}} i\right] T_{S F, j}+s_{m n}
\end{aligned}
$$

where, $F_{i, j}$ and $T_{i, j}$ are the maximum force and time to peak force of the $i_{t h} \mathrm{MU}$, respectively. $F_{S F, j}$ and $T_{S F, j}$ are scaling factors that determine the parameters of individual MUs. $p_{m x}$, $p_{m n}, s_{m n}$ and $s_{s l}$ are constants that determine the shape of the distribution and are defined in Table I. As reported in human physiology [18], [22], muscle fibre bundles described by (9) and (10) were uniformly distributed within all heads of the corresponding muscle. Passive resistance from synovial fluid (viscous fluid between moving bony surfaces), tendons and wrapping ligaments were modeled as a resistive torque $r^{-}$proportional to the joint velocity $\dot{q}$ :

$$
r^{-}=\dot{q} r_{S F}
$$

where, the scaling factor $r_{S F}$ was determined in the identification process.

\section{Sensitivity Analysis}

We conducted a sensitivity analysis on the combined NMS model. In order to check the necessary conditions of identifiability, we computed the output variances by changing the model parameters. Sensitivity to parameter changes were calculated using the measure, $G$

$$
G=\sum_{p=1}^{6} \frac{\sum_{t=0}^{2}\left(x_{p}(t)-\hat{x}_{p}(t)\right)^{2}}{\sum_{t=0}^{2}\left(x_{p}(t)\right)^{2}}
$$

where $x_{p}(t)$ is the $p$ th output at time step $t$ in the forward dynamics computations. $\hat{x}_{p}(t)$ refers to the output of the system for a nominal set of parameters and input variables. The outer summation $p=1 . .6$ accumulates the output difference over joint angle $q$, joint torque $r$, muscle forces $f_{B}$ and $f_{T}$, and activations $a_{B}$ and $a_{T}$.

We applied changes to each of the parameters in steps of: $0.1,0.25,0.5,1.0,2.0$ times the nominal value, and computed the sensitivity measure $G$. Fig. 3 plots the results for some of the most sensitive parameters. In general the system showed good excitability to modulation of the MU parameters such as $T_{S F, j}$ and $F_{S F, j}$. The output also showed high sensitivity to parameters that modulate neuron soma diameter (and hence the firing pattern) $D_{S F, j}$. The lowest sensitivities were found for some of the reflex parameters, e.g., $\omega_{B I+}, \omega_{T T+}, \sigma_{I T-}$. Note that in our analysis we modified each of the parameters one at 


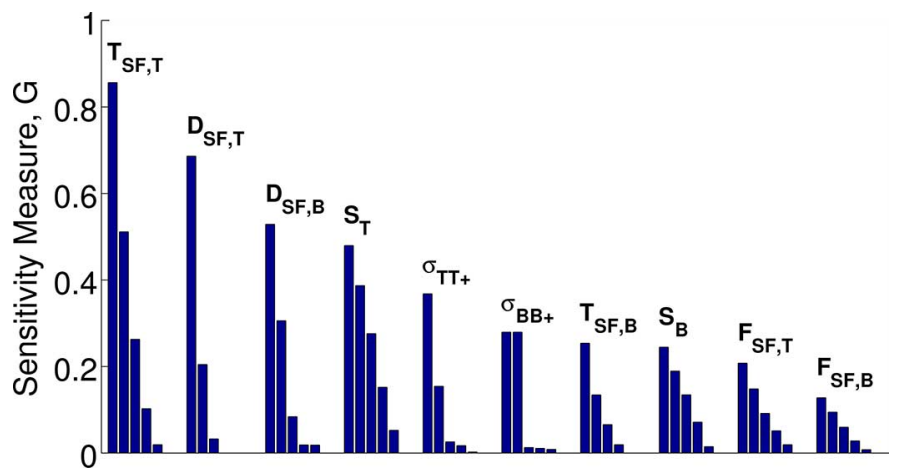

Fig. 3. Sensivity Analysis: Bar plots in descending order show the sensivity measure $\mathrm{G}((12))$ computed by varying model parameters and inputs. Grouped bars for each parameter show $\mathrm{G}$ for different perturbation magnitudes (left to right $-2.0 x, 1.0 x, 0.5 x, 0.25 x, 0.1 x)$. Missing bars indicate that the sensitivity measure could not be computed for that parameter set due to output instability.

a time, but not for all possible combinations, i.e., we did not cover the entire plausible parameter space. It is possible that the some of low sensitivities observed about the currently chosen nominal values are not present in other reaches of the parameter space.

\section{HUMAN EXPERIMENTS}

Two conditions, Graded Recruitment (GR) and Co-contracted Reflex (CR), were designed to identify the parameters of the NMS model. A third condition, Loaded Reflex (LR), was used to verify the results of the identified model.

\section{A. Participants}

Five male volunteers took part in the experiments. Mean demographics \pm variance: age, $25.6 \pm 3.2$ years, weight, $64.6 \pm$ $11.6 \mathrm{~kg}$, height, $178.8 \pm 9.3 \mathrm{~cm}$. Participants were in good health and did not report any neurological or physiological conditions that might interfere with the experiments. They were all right-handed and chose to use the right arm for the experiments. Informed written consent was obtained prior to the experiments. The protocol was designed in accordance with the ethical standards of the Declaration of Helsinki (rev. 2013).

\section{B. Materials and Methods}

Participant joint position data were collected with a 10-camera motion capture system (MotionAnalysis Co., USA) by attaching markers to the shoulder, elbow and wrist lateral and medial protuberances, and the dorsal surface of the hand. The resolution of the capture volume was about $3 \mathrm{~mm}$, and data was recorded at $200 \mathrm{~Hz}$. Three wireless electromyographs (Delsys Inc., USA) were attached to the skin by palpitating the mid-point of the biceps muscle, and the lateral and medial heads of the triceps muscle. In some trials, we used a bi-directional force sensor (Imada Z2-1 J, Range $\pm 500 \mathrm{~N}$, Resolution $0.001 \mathrm{~N}$ ), mounted on a pedestal such that the sensor axis was perpendicular to the floor. The sensor could be moved vertically to adjust for participant height and the mounting apparatus was heavy enough to be immovable by the participant. We built a strap made up of a cushion and a rigid element that could be worn around the wrist. The rigid element could be fixed on to the sensor such that a downward or upward force could be applied along the sensor axis by extending or flexing the elbow, respectively. Both EMG and force sensor data were recorded at time-matched instances at $1000 \mathrm{~Hz}$. EMG signals were high-pass filtered at $30 \mathrm{~Hz}$, rectified, and then low pass filtered at $20 \mathrm{~Hz}$. Force sensor and motion capture position data were both low pass filtered at $20 \mathrm{~Hz}$. We used second-order zero phase delay Butterworth filters in all cases. For all conditions, participants were asked to position their arm such that the upper arm was vertical and the forearm horizontal (i.e., extending a $90^{\circ}$ angle at the elbow) and supinated.

In condition 1, GR, the force sensor was positioned such that sensor axis was directly below the participant's wrist. After attaching the wrist strap, we displayed the current force being exerted by the participant on the sensor as a real-time readout on a monitor in front of them. Participants were asked to only apply force by flexion/extension at the elbow, and not for example by moving their torso or applying their body weight on the sensor. Compliance with this instruction was verified visually during the practice trials, and post-hoc using the variance in shoulder markers. Trial conditions consisted of asking the participants to apply forces of $-50 \mathrm{~N},-30 \mathrm{~N},-10 \mathrm{~N}$ (pushing downwards) and $+10 \mathrm{~N},+30 \mathrm{~N},+50 \mathrm{~N}$ (pulling upwards) on the sensor. Conditions were presented in randomized order. Trials began with an audible cue signalling that the participant should apply the requested force level, and maintain it for $10 \mathrm{~s}$. A second audible cue signalled the end of the trial. Trials were separated by gaps of 2 min to minimize effects of fatigue.

In condition 2, CR, participants were asked to co-contract the biceps and triceps muscles to three subjective levels; Low Co-contraction (LC), Medium Co-contraction (MC) and High Co-contraction (HC). Note that the force sensor and pedestal were removed from the experimental area for these trials. Participants wore headphones that played an audible cue to signal the start of trial, and then white noise to mask laboratory sounds. In addition, a blindfold was drawn over the participant's eyes. They then co-contracted the biceps and triceps of the instrumented arm to the requested subjective level. At a randomly chosen instant between 5-20 s from start of trial, the experimenter applied a sudden perturbation to the participant's wrist. Perturbation was in the form of a falling weight released from about $0.5 \mathrm{~m}$ above the wrist. The weight was a small plastic cylinder ( $15 \mathrm{~cm}$ length $\times 5 \mathrm{~cm}$ diameter) weighing $0.5 \mathrm{~kg}$. The motion of the weight was tracked with motion capture markers to detect the exact start of perturbation and height of fall. Trials ended about 1-2 $\mathrm{s}$ after perturbation was detected by the participant. Trials were separated by $2 \mathrm{~min}$ to minimize effects of fatigue. In pilot trials, the joint velocity resulting from this perturbation was recorded between $200-300^{\circ} / \mathrm{s}$, which was above the $175^{\circ} / \mathrm{s}$ threshold reported for inducing the stretch reflex [32].

We recorded a third condition, LR, which was used for model verification. Here, we loaded the forearm with 1,3 , or $5 \mathrm{~kg}$ weights. Similar to the CR condition, a randomly timed perturbation was applied to the wrist. Recording sessions began after all participants practised a few trials from the GR, CR and LR conditions. They all reported that the conditions were easy to follow and that the perturbation force in CR condition was not uncomfortable. In addition, we recorded the subject-specific Maximum Voluntary Contraction (MVC) information be- 
fore the start of experiments. These recordings were done in order to estimate the maximum muscle forces for the biceps and triceps muscle, and the corresponding maximum sensor values of the EMG electrodes. The methodology was similar to the GR experiment, participants were asked to apply the maximum possible force on the sensor by flexing or extending the elbow and hold it for $5 \mathrm{~s}$. The maximum value during these trials was chosen as the MVC. The entire experiment, per participant, was recorded in one session of about 1 to $1.5 \mathrm{~h}$ with gaps of 5-10 min between conditions.

\section{Inverse Dynamics Analysis}

Musculoskeletal Inverse Dynamics (ID) was calculated using a custom-built software, sDIMS [16]. The skeletal and muscular kinematics were identical to that defined in Section II-C. The muscle dynamics were described by Hill-type models (8), using $F$ values estimated from the MVC recordings. Note that in the inverse dynamics estimation, $F$ denotes the total force being generated by the whole muscle (in contrast to the motor units maximum forces, $F_{i, j}$, used in the forward dynamics computations). An accurate value of $F$ was vital to the estimation of the muscle force magnitudes. After filtering of force sensor data, the highest recorded value during MVC trials and the lever arms of the biceps and triceps muscle, were used to compute the maximum muscle force. The lever arms were computed from the scaled subject-specific musculoskeletal kinematics. Based on these values we approximated the subject-specific $F$ values, with mean $\pm \mathrm{SD}=1121.8 \pm 67.7 \mathrm{~N}$ for the biceps, and, $1247.8 \pm 257.5 \mathrm{~N}$ for the triceps. The generalized forces $r_{G}$ were then computed as

with

$$
r_{G}=J^{T} f+J_{C}^{T} r_{C}
$$

$$
J=\frac{\partial l}{\partial q}
$$

where, $J$ is the Jacobian matrix of wire lengths with respect to generalized coordinates, $f$ are the muscle tensions, $J_{C}$ is the Jacobian matrix of contact points with respect to generalized coordinates and $r_{C}$ the contact forces [16]. To compute the muscle tensions at each time step, an optimization problem was defined with constraints coming from the Hill-type force estimation, terms to minimize overall muscle forces, and terms to ensure continuity of estimated values. For the Hill-type computation, the activation levels $a(t)$ were estimated based on EMG recordings and the corresponding subject-specific $F$ value. This optimization problem was then solved using quadratic programming [16], [33].

External contact forces such as that exerted upon the sensor in the GR condition, or, the perturbation in the CR experiment were applied to the wrist joint of the model. For the GR condition, exerted force could be directly obtained from the force sensor readings. For the CR condition, we estimated the perturbation force using the weight of the object and post-hoc analysis of the velocity profile. From the velocity profile and the video recordings we observed that the contact between object and wrist lasted approximately $300 \mathrm{~ms}$. For this duration, the profile of the perturbation force was approximated as a symmetric exponential rise and fall.

\section{Reference Data}

The results of the musculoskeletal ID analysis were used as the reference for parameter identification. For the GR condition, the reference data consisted of

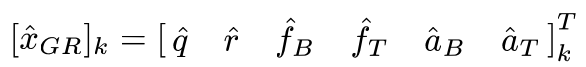

where, $\hat{q}$ was the joint angle, $\hat{r}$ the joint torque, $\hat{f}_{B}, \hat{f}_{T}$ the biceps and triceps muscle forces, and $\hat{a}_{B}, \hat{a}_{T}$ the corresponding muscle activations. Here, $\hat{f}_{B}$ and $\hat{f}_{T}$ were computed as the cumulative force generated by all heads of the biceps and triceps. Note that the reference was calculated as the average results over the duration of each GR condition, after the required force level was achieved. The reference values in (14) were calculated for each participant and for $k=1 . .6 \mathrm{GR}$ recruitment levels.

For the CR condition, we similarly calculated reference values for the three co-contraction levels. In contrast to the time-averaged values of the GR reference, here the reference was a trajectory over time of the parameters in (14), i.e.,

$$
\left[\hat{x}_{C R}\right]_{k}=\left[\begin{array}{llllll}
\hat{q}(t) & \hat{r}(t) & \hat{f}_{B}(t) & \hat{f}_{T}(t) & \hat{a}_{B}(t) & \hat{a}_{T}(t)
\end{array}\right]_{k}^{T}
$$

with, $t=0-125 \mathrm{~ms}$. The reference time window was chosen to be $25 \mathrm{~ms}$ before, to $100 \mathrm{~ms}$ after the perturbation. Fig. 5 plots the computed ID reference data as empty circles.

\section{PARAMETER IDENTIFICATION}

In Section II some of the model parameters were selected and fixed based on literature evidence, for example the number of MUs. Others were distributed over a range based on known physiological relationships, with their exact values to be determined by subject-specific scale factors (for example, the distribution of MU $F_{i, j}$ and $T_{i, j}$ ). Here, we describe the identification process for these unknown parameters from the experimental data.

\section{A. Forward Dynamics Simulation}

We recall that the control variables for the NMS model described in Section II were the biceps and triceps stimulations, $S_{B}$ and $S_{T}$, respectively. Based on our neural architecture a higher stimulation would result in a higher number of motor neurons firing, and consequently a larger force being generated by the corresponding muscle fibres. For a given stimulation of the biceps and triceps MN pools, the behavior of the NMS model was then calculated using a fourth-order Runge-Kutta algorithm with an integration time step of $0.5 \mathrm{~ms}$. Simulation duration was $6 \mathrm{~s}$, with data from the first $4 \mathrm{~s}$ not being considered for identification. This allowed sufficient time for the NMS dynamics to stabilize in the absence of external perturbation. Computations for a single simulation took about $30 \mathrm{~s}$ on a $2 \times 2.3$ Ghz computing node, and multiple instances were highly parallelized using the OpenMPI framework. Parameter identification problem was solved using the derivative free method BOBYQA (Bound Optimization BY Quadratic Approximation) [34], implemented in the NLopt library [35]. To simulate the GR and 

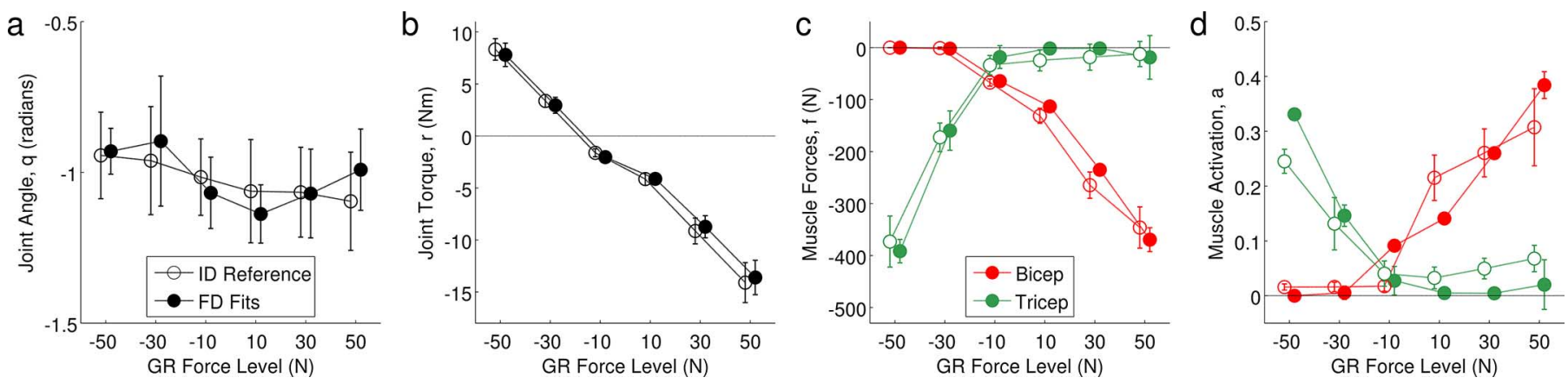

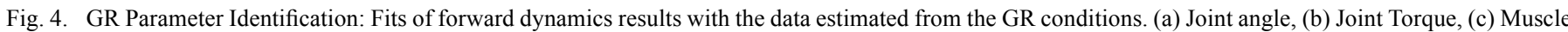
forces and (d) Muscle activations. Error bars indicate standard deviations of the mean across participants.

CR conditions, identical external forces as those used in the ID computations were applied to the NMS model.

\section{B. Isometric Parameters}

By the term Isometric we refer to the asymptotic response of the NMS model under a constant stimulation to the biceps and triceps neural pools, and in the absence of any other perturbation. We indicate the following parameters that contribute to this response, $\left[D_{S F, B}, D_{S F, T}, F_{S F, B}, F_{S F, T}\right]$ and the control inputs $\left[S_{B}, S_{T}\right]$. These parameters were identified from the GR reference data (14) by solving the least-squares objective function

$$
\min \cdot \sum_{k=1}^{6}\left\|W\left(\left[x_{G R}\right]_{k}-\left[\hat{x}_{G R}\right]_{k}\right)\right\|^{2}
$$

with,

$$
z_{G R}=\left[\begin{array}{llll}
D_{S F, B} & D_{S F, T} & F_{S F, B} & F_{S F, T} \\
\left\{S_{B}\right. & \left.S_{T}\right\}_{1 \ldots k}
\end{array}\right]
$$

where, $k=1 . .6$ refers to the applied force levels in the GR condition. $x_{G R}$ was the vector of reference values from (14) and $\hat{x}_{G R}$ the steady state estimates from the forward dynamics simulation. $W$ was a diagonal matrix of normalizing factors that adjusted the variables to the same order of magnitude. These normalizing factors were determined post-hoc as the mean of the reference values $x_{G R} . z_{G R}$ refers to the isometric parameters to be identified. $S_{B_{-} 1 \ldots k}$ and $S_{T_{-1} \ldots k}$ were the 6 control inputs (neural stimulations) to be determined, corresponding to the $6 \mathrm{GR}$ levels. The problem thus consisted of solving for the 16 parameters, four of which were common to all GR levels and the other $6 \times 2$ parameters specific to the six GR recruitment levels.

The identification was constrained to values of free parameters $z_{G R}$ that resulted in physiologically realistic values for membrane capacitances, time constants and motor unit twitch forces (Table I). For these computations, the remaining dynamic NMS parameters were fixed at the means of the full physiological range. For each participant we first evaluated the value of (16) at 1000 points randomly distributed among the parameters in (17). We then ran the identification problem for $z_{G R}$ combinations with the 10 lowest costs to ensure that the parameter envelope defined by (17) was well explored.

\section{Dynamic Parameters}

We classify the remaining parameters as pertaining to the dynamic response of the NMS model which were identified using the CR reference data. To account for a trajectory of reference values (15), we modified the minimization problem as

$$
\begin{gathered}
\min \cdot \sum_{k=1}^{3}\left\|W \sum_{t=0}^{125}\left(\left[x_{C R}\right]_{k}(t)-\left[\hat{x}_{C R}\right]_{k}(t)\right)\right\|^{2} \\
\text { with, } z_{C R}=\left[\begin{array}{ccc}
T_{S F, B} & T_{S F, T} & r_{S F} \\
\left\{S_{B}\right. & \left.S_{T}\right\}_{1 \ldots k} & \\
\left\{w_{i j \pm}\right. & \left.\sigma_{i j \pm}\right\} &
\end{array}\right]
\end{gathered}
$$

where $k=1,2,3$, refers to the co-contraction levels $[L C, M C, H C]$ of the $\mathrm{CR}$ condition. Here, the objective function (18) minimizes the error between the forward dynamics estimate, $\hat{x}_{C R}$ and the reference $x_{C R}$, for $t=0$ to $125 \mathrm{~ms}$, with the free model parameters $z_{C R}$ subject to the bounds in Table I. Here, the first 3 parameters in $z_{C R}$ were common to all CR levels, and the other 30 were specific to each $\mathrm{CR}$ level (10 per CR level). In other words, the parameter identification problem was designed such that stimulation and reflex feedback could vary between the three recorded CR levels. Similar to GR identification, the value of the objective function (18) was evaluated at 6000 random points in the parameter space and the identification process launched from 10 of the points with the lowest costs.

\section{Identification Results}

In general the identification process provided a good fit between the forward dynamics estimation and the reference data. Figs. 4 and 5 plot the fits obtained between the forward dynamics behavior and the inverse dynamics reference for the GR and CR conditions, respectively. The average differences for GR conditions (across all force levels and all participants) in joint angle, joint torque, muscle forces and muscle activations were: $0.11 \pm 0.06$ radians, $0.5 \pm 0.16 \mathrm{Nm}, 17.5 \pm 18.9 \mathrm{~N}$, and $0.025 \pm 0.016$, respectively. The corresponding differences for the $\mathrm{CR}$ conditions were: $0.05 \pm 0.04$ radians, $1.1 \pm 1.12 \mathrm{Nm}$, $21.1 \pm 14.3 \mathrm{~N}$, and $0.04 \pm 0.01$. Under isometric conditions, the joint angle, joint torque, cumulative muscle forces and muscle activation levels from forward dynamics closely matched those calculated from the inverse dynamics computations. This may be observed from the fits in Fig. 4 as well as the values in Fig. 5 before perturbation was applied. For the dynamic behavior of the model after perturbation, the largest discrepancy between inverse and forward dynamics was observed in joint angle. The FD model presented with excessive joint extension 

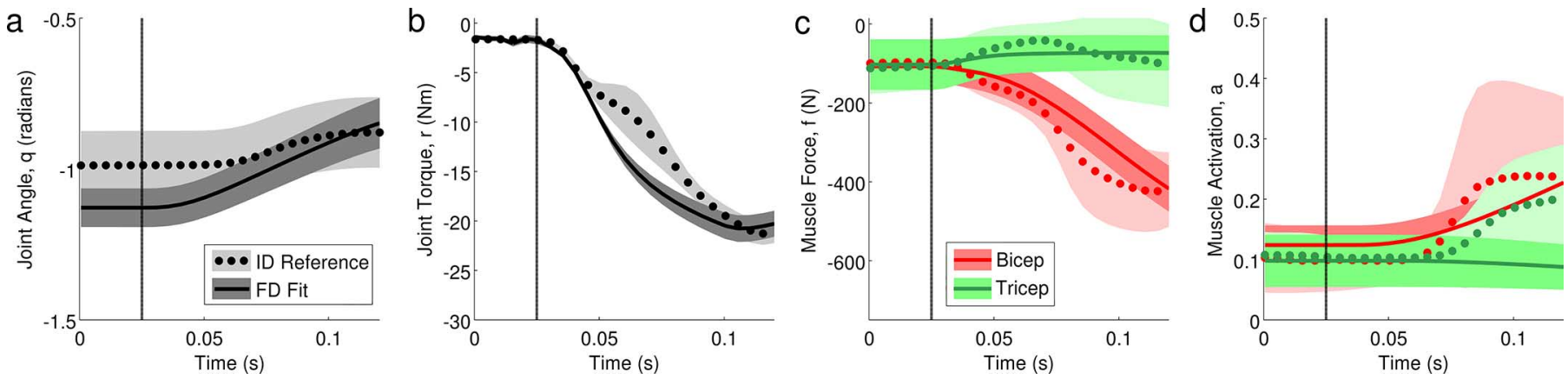

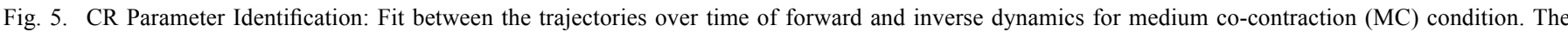
vertical line indicates the time instant when the arm was perturbed. Shaded regions indicate the standard deviation across participants.
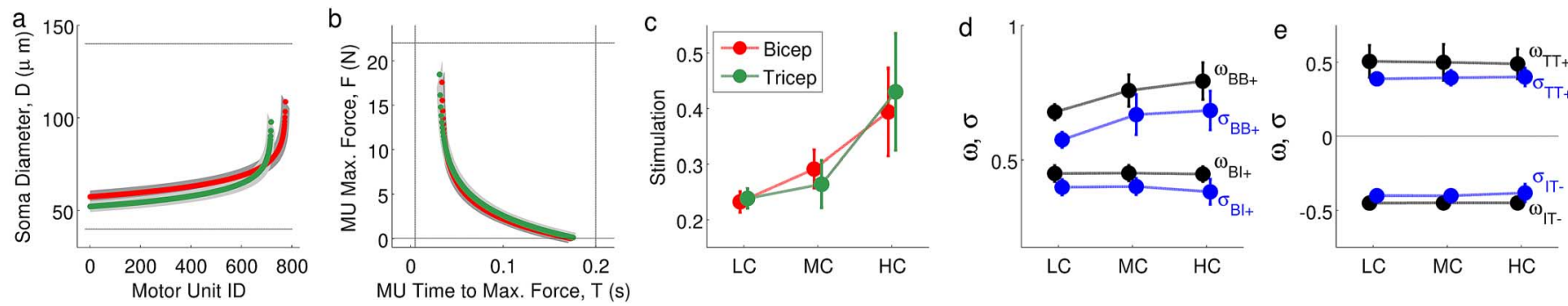

Fig. 6. Identification Results: (a) Variation in soma diameters computed as per (2) and the identification parameter $D_{S F, j}$. Shaded region indicates the standard deviation of the mean across participants. Thin lines indicate the identification search range. (b) Distribution of biceps and triceps motor unit $F$ and $T$ values over all participants. (c), (d) and (e) Normalized stimulation and synaptic weights for the various neural pools. Black circles indicate the center weight term of the Gaussian distribution $\omega$, and blue circles the corresponding variance $\sigma$. Error bars indicate the standard deviation of the mean across participants.

after perturbation, Fig. 5(a), possibly due to the absence of other wrapping muscles and tendons in the NMS model.

Fig. 6 and Table I show the range of identified physiological and normalized parameters for all participants. Statistical significance of results was tested in SPSS 22.0 (IBM Corp., USA). Significance level was set at a p-value of 0.05 . Mauchly's test was used to confirm the assumption of sphericity, and in cases of violation we applied the Greenhouse-Geisser correction and report the corrected degrees of freedom. Motor neuron soma diameters ranged between $57.08 \mu \mathrm{m}$ to $109.37 \mu \mathrm{m}$, Fig. 6(a). Across participants, the corresponding identification parameter $D_{S F, j}$ showed a variation of upto $13.8 \%$ over the mean. $F_{S F, j}$ and $T_{S F, j}$ varied by $19.6 \%$ and $13.7 \%$ across participants, respectively. The resulting range in motor unit maximum twitch force and time was from $0.0124 \mathrm{~N}$ to $20.2 \mathrm{~N}$, and $28.2 \mathrm{~ms}$ to 179 ms, respectively [Fig. 6(b)]. $\tau_{S F}$ ranged from 0.578 to 0.756 with mean $\pm \mathrm{SD}=0.7074 \pm 0.0756$.

Fig. 6(c) and (d) plots the identified stimulation and synaptic weights between all neural pools, for the CR conditions. As expected, the identified stimulation values to the biceps and triceps motor neuron pools increased for higher co-contraction conditions. This increase was significant across participants for both biceps and triceps stimulation, $F(2,8)=16.68, p<$ $0.001, \eta^{2}=0.807 \& F(1.07,4.281)=20.113, p=0.009$, $\eta^{2}=0.834$. Interestingly, the parameters responsible for monosynaptic excitations between the biceps MS to biceps MN (positive feedback), $w_{B B+}$ and $\sigma_{B B+}$, also showed a significant increase with co-contraction level, $F(2,8)=9.236, p=0.008$, $\eta^{2}=0.698$ for $\omega_{B B+}$ and $F(2,8)=6.255, p=0.023$, $\eta^{2}=0.61$ for $\sigma_{B B+}$. In comparison, triceps mono-synaptic excitation values, $w_{T T+}$ and $\sigma_{T T+}$ were smaller and of sim- ilar magnitude to the weights for di-synaptic inhibition, $w_{B I+}$, $\sigma_{B I+}$ and $w_{I T-}, \sigma_{I T-}$, and showed no significant variation across conditions (all $p^{\prime} s>0.05$ ). Recall that synaptic characteristics were derived from a distance-dependent Gaussian distribution (Section II-A). Based on the identified values we found that source neurons made substantial connections (above 10\% of center weight $\omega$ ) with about $95 \%$ of target neurons for the largest $\sigma$, to about $45 \%$ for the smallest $\sigma$.

\section{E. Model Verification}

The identified models were tested by comparing them to the inverse dynamics results from the LR condition. In order to simulate the LR conditions in the forward dynamics model, we manually chose the control inputs $S_{B}$, such that the elbow angle matched the observation before the application of perturbation. Triceps stimulations, $S_{T}$, were set to zero. Note that in the LR experiment the wrist was progressively loaded with heavier weights (W1, W3, and W5). Thus, in order to maintain the forearm posture the biceps motor neurons required higher and higher stimulations. As we found a dependency between the stimulation and neural feedback parameters, we chose to simulate the [W1,W3,W5] conditions of the LR experiment with the neural weights identified for the [LC,MC,HC] conditions of the CR experiment, respectively. We observed that the identified models could predict the initial states of joint angle, joint torque, muscle forces and activations quite well. However, the dynamic behavior after perturbation showed larger differences for predicted muscle forces and activations than those observed in the CR fits. The differences in joint angle, joint torque, muscle forces and muscle activations were: (mean \pm variance, over all 
a $50 \%$ Feedback

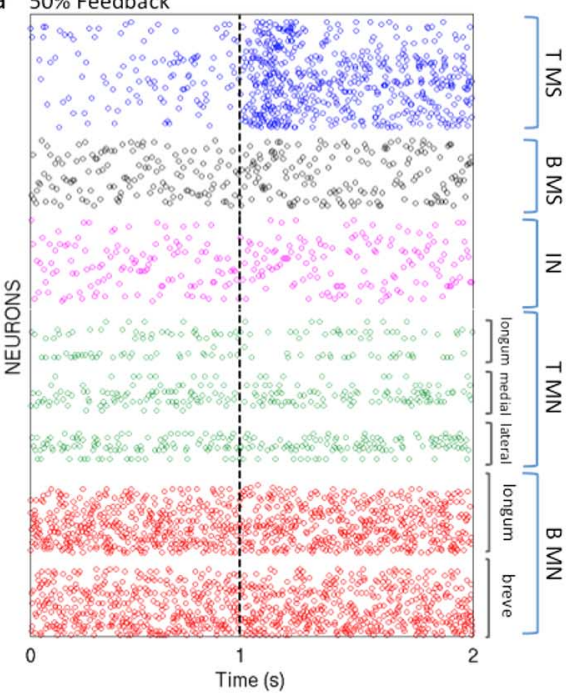

b $100 \%$ Feedback

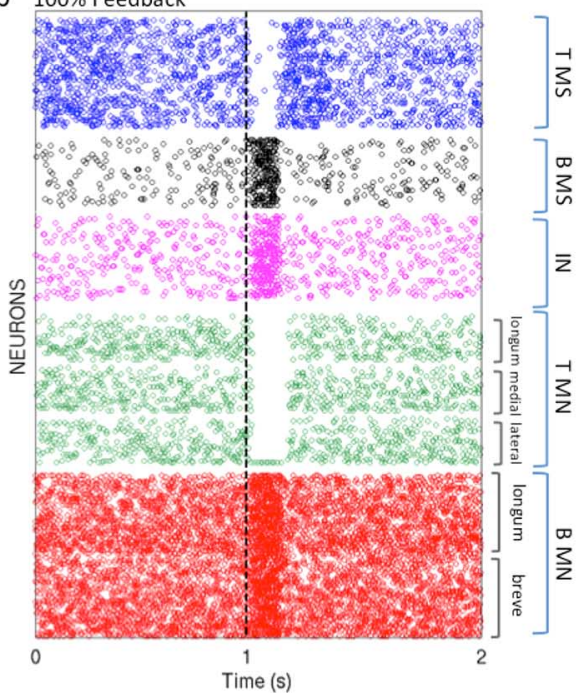

C $150 \%$ Feedback

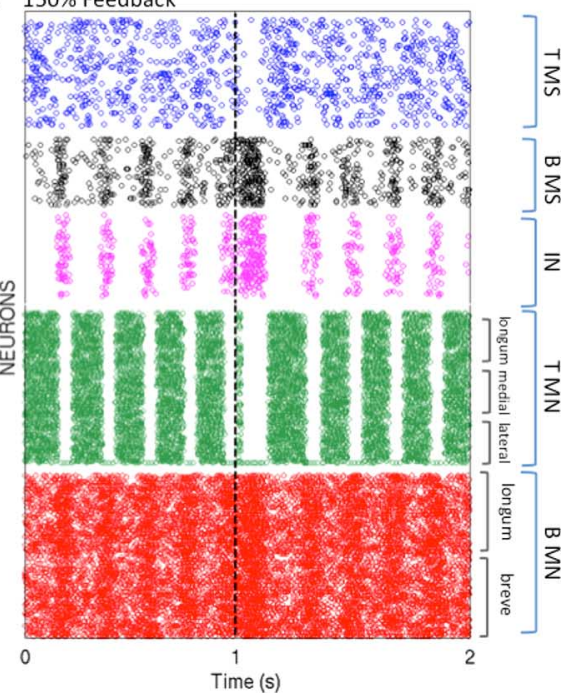

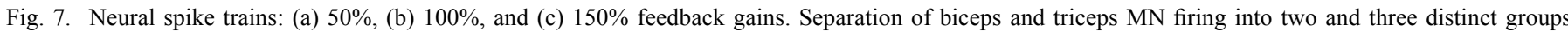

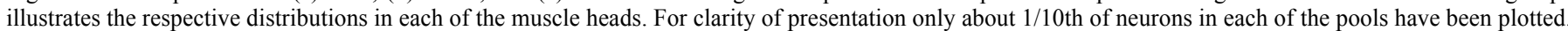

conditions and all participants): $0.04 \pm 0.03$ radians, $0.16 \pm$ $0.08 \mathrm{Nm}, 11.1 \pm 2.8 \mathrm{~N}$, and $0.03 \pm 0.005$.

\section{Simulation of the Stretch RefleX}

Fig. 7 plots simulated spike trains under varying feedback characteristics. We applied a constant stimulation to the biceps and triceps MN pools such that around $25 \%$ of MNs were recruited in each pool. To approximate varying reflex sensitivity we scaled the synaptic parameters, $\omega_{B B+}, \sigma_{B B+}$ and $\omega_{T T+}$, $\sigma_{T T+}$, from $50 \%$ to $150 \%$ of the values identified for Sub3 - W3 condition, reflecting weak to excessive feedback. A perturbation was applied to the wrist after $1 s$. For low and $100 \%$ feedback the NMS model exhibited stable settling behavior before and after the perturbation. We observed that for $50 \%$ feedback gain condition there was little muscle force generated due to the stretch perturbation. This relative unresponsiveness of the system can also be observed in the almost uniform MN and IN spike trains in Fig. 7(a). At 100\% feedback the perturbation had a significant effect on the NMS dynamics. The spike trains in Fig. 7(b) show that the biceps stretch results in increased biceps MN firing and inhibition of triceps MN firing. This resulted in larger biceps muscle forces and further inhibition of triceps muscle forces. As feedback gains were increased to $150 \%$, the system show rhythmic self-excitatory behavior at about $5 \mathrm{~Hz}$, even without external perturbation, Fig. 7(c). In physiology, such rhythmic contraction/relaxation of muscle is characterized as clonus and a hyperactive stretch reflex has been suggested to be one of its main causes [2], [5], [32].

\section{DISCUSSION}

In this study we developed a neuromusculoskeletal model of the stretch reflex in the human arm, and identified subject-specific parameters from experimental data. The model included the dynamics of spiking motor neurons and muscle fibre bundles (motor units), Ia spindle afferents, intra-spinal connections and skeletal mechanics. The identified model was able to track the recorded reflex behavior (joint positions), as well as the estimated joint torques, and muscle forces and activations. The exhibited spike patterns were consistent with those one may expect from literature evidence [22], [32].

The implementation of discrete neural control layers came at the cost of additional model parameters that had to be determined. The challenges and added complexity of this problem may be justified in three ways. First, the physiologically realistic neuromuscular architecture allows for the investigation of phenomena such as recruitment and rate coding [18], [22]. This would not have been possible if the neural control were to be simplified as a scalar muscle activation ratio. Second, a biomimetic control architecture is a step towards such models being more clinically relevant (although in the present case our model simplifications limit this application). For such models, identifying the model properties also helps in inferring neurophysiological states that are not directly observable. This is an advantage when designing neural interfaces for rehabilitation, for example neuro-prostheses, or when estimating normal or pathological neuromuscular behavior. Third, by designing the connections between discrete spinal networks, continuous muscle force generation and skeletal movements, such models are the first step towards connecting physical movements to the motor cortex and voluntary control.

The identified models produced a neural-reflex (approximate start of mono-synaptic excitations of agonist MNs) about $15 \mathrm{~ms}$, and a muscular reflex 25-50 ms (agonist muscle force increase), after perturbation. The realism of the neural-reflex, number of MUs recruited, was difficult to evaluate due to the lack of human experimental data on individual motor unit recordings. However, the resultant muscle forces and joint torques were consistent in magnitude and rate of increase with those typically reported in human movement studies [10], [11], [36]. We also found that the neuromuscular model parameters compare well with results previously reported in literature. The identified MN 
membrane capacitances and time constants (based on soma diameter range, $50.83 \mu \mathrm{m}$ to $109.37 \mu \mathrm{m}$ ) were similar to those reported by Cisi and Kohn [11] and Fleshman et al. [23], as were the motor unit contraction times ( $28 \mathrm{~ms}$ to $179 \mathrm{~ms}$ ). Motor unit twitch forces were much higher than those typically reported [11], [28]. However, this was to be expected to take into account the higher force generation capabilities of large human muscles compared to the smaller animal muscles that are typically tested. We also found an increase in monosynaptic excitatory feedback with higher muscle co-contractions. This phenomenon has been previously reported to be related to reflex modulation via gamma-motorneuron activity, and suggested to occur as a function of task, co-contraction and perturbation characteristics [3], [5], [6], [10], [32]. However, as our spindle model did not contain any fusimotor components, this particular effect could not be further investigated. As reflex modulation can also play an important role in the control of joint stiffness, an alternative would be to incorporate a more complex spindle model (e.g., [24]).

As mentioned earlier, the focus of this study was on the identification of neuromuscular parameters at the motor unit level while incorporating the effects of skeletal movements, neural and mechanical feedback. For this reason we simplified the neural, muscular and skeletal components - and as is the case with every model, the underlying assumptions and simplifications limit the prediction capabilities. At the neural level the most significant effects were the missing internal pools and feedback loops related to the Ib, II afferents, sensitivity tuning by gamma-motorneurons, as well as axonal and dendritic effects [9], [11], [24]. These components play an important role in modulating reflexes and form a major part in organizing involuntary behaviors. Due to our model simplifications we constrain our analysis to isometric conditions and movements occurring within $100 \mathrm{~ms}$ after perturbation. The assumption here was that movements within this time window are not affected by tuning mechanisms, slower afferents or voluntary control. An additional limitation arises from the simplified muscle model that accounts for the macroscopic mechanical behavior, but not detailed electrochemical behavior such as those tackled in continuum mechanics models (see, for example, [13]). The absence of other arm muscles such as the brachioradialis and wrapping ligaments, may have been one of the causes for excessive extension exhibited by our model. Due to the same reason, there may also have been an overestimation of biceps and triceps forces. We assumed uniform distributions of the neuromuscular parameters based on literature evidence. Most of these studies report data from animal experiments, or cadaveric observations, and the extent to which they reflect healthy human neurophysiology is presently unclear. In order to improve the ecological usefulness of our current approach, it would be necessary to include these missing components. In this context, the contribution of our study is to provide the basis for these extensions and help counter potential problems in a larger scale NMS model (e.g., by pre-identification of the presently included component parameters).

Finally, identification based on optimization alone cannot tackle the problem of neurophysiological redundancy. For example, the increase in mono-synaptic reflex gains could be due to a fusimotor tuning based higher sensitivity of the muscle spindles. However, the same behavior (excessive firing of agonist motor neurons) could also be exhibited if we were to decrease the motor neuron firing threshold [2]. To solve this redundancy we have to introduce additional measurements that can help discriminate between competing solutions. In human neurophysiology in vivo spinal recording is presently unrealistic. However, new advances in EMG technology offer possible solutions such as dense electrode-matrices and associated algorithms that can decompose individual motor unit action potentials (MUAPs) [37]. This decomposition may help achieve a more detailed observation of the neurophysiological state, and consequently a better estimate in the parameter identification stage.

Despite these limitations, we assert that the architecture and identification methodology proposed in this study have useful applications, are modular and extendable to more complex neuromuscular behavior. For example, in order to complete the spinal reflex circuits as proposed by Bashor [9], our model requires additional neural pools and connections. The design of these pools is relatively straight-forward, however, one challenge would be to design experimental paradigms to identify these (additional) parameters. Due to the inherent redundancy in human neuromuscular control, a careful choice of the reference data used in parameter identification as well as the algorithms used would be vital. Similarly, the extension of the current agonist-antagonist framework to include more limbs would be technically straight-forward. It is however not obvious how more complicated muscle actions that do not directly act as agonist or antagonist, or those that change function with movements, may be included in our framework. Recent advancements in muscle primitives may prove to be a valuable asset here [7]. We may consider to develop the concept of neural primitives that denote descending drives from the upper spinal levels, and would serve to modulate and coordinate the behavior of many interconnected pools of spinal neurons.

\section{ACKNOWLEDGMENT}

The authors thank the Interdisciplinary Center for Scientific Computing, University of Heidelberg for use of their computational facilities; and A. Murai, J. Moren, K. Mombaur and D. Clever for helpful discussions.

\section{REFERENCES}

[1] A. K. Silverman and R. R. Neptune, "Muscle and prosthesis contributions to amputee walking mechanics: A modeling study," J. Biomech., vol. 45, no. 13, pp. 2271-2278, Aug. 2012.

[2] J. M. Hidler and W. Z. Rymer, "A simulation study of reflex instability in spasticity: Origins of clonus," IEEE Trans. Rehabil. Eng., vol. 7, no. 3, pp. 327-340, Sep. 1999.

[3] A. C. Schouten, E. de Vlugt, J. B. van Hilten, and F. C. van der Helm, "Quantifying proprioceptive reflexes during position control of the human arm," IEEE Trans. Biomed. Eng., vol. 55, no. 1, pp. 311-321, Jan. 2008.

[4] J. Schuurmans, F. C. van der Helm, and A. C. Schouten, "Relating reflex gain modulation in posture control to underlying neural network properties using a neuromusculoskeletal model," J. Comput. Neurosci., vol. 30, no. 3, pp. 555-565, Jun. 2011.

[5] E. de Vlugt, J. H. de Groot, W. H. Wisman, and C. G. Meskers, "Clonus is explained from increased reflex gain and enlarged tissue viscoelasticity," J. Biomech., vol. 45, no. 1, pp. 148-155, Jan. 2012. 
[6] W. Mugge, A. G. Munts, A. C. Schouten, and F. C. van der Helm, "Modeling movement disorders-CRPS-related dystonia explained by abnormal proprioceptive reflexes," J. Biomech., vol. 45, no. 1, pp. 90-98, Jan. 2012.

[7] M. Sartori, L. Gizzi, D. G. Lloyd, and D. Farina, "A musculoskeletal model of human locomotion driven by a low dimensional set of impulsive excitation primitives," Front. Comput. Neurosci., vol. 7, no. 79, 2013.

[8] E. Henneman and C. Olson, "Relations between structure and function in the design of skeletal muscle," J. Neurophysiol., vol. 28, pp. 581-598, 1965.

[9] D. P. Bashor, "A large-scale model of some spinal reflex circuits," Biol. Cybern., vol. 78, no. 2, pp. 147-157, Feb. 1998.

[10] A. H. A. Stienen, A. C. Schouten, J. Schuurmans, and F. C. T. van der Helm, "Analysis of reflex modulation with a biologically realistic neural network," J. Comput. Neurosci., vol. 23, no. 3, pp. 333-348, Dec. 2007.

[11] R. R. L. Cisi and A. F. Kohn, "Simulation system of spinal cord motor nuclei and associated nerves and muscles, in a web-based architecture," J. Comput. Neurosci., vol. 25 , no. 3, pp. 520-542, Dec. 2008.

[12] M. Sreenivasa, A. Murai, and Y. Nakamura, "Modeling and identification of the human arm stretch reflex using a realistic spiking neural network and musculoskeletal model," in IEEE/RSJ Int. C Int. Robot (IROS), 2013, pp. 329-334.

[13] T. Heidlauf, F. Negro, D. Farina, and O. Röhrle, "An integrated model of the neuromuscular system," in 6th Int. IEEE/EMBS C Neural Eng. (NER), 2013, pp. 227-230.

[14] A. J. Fuglevand, D. Winter, and A. E. Patla, "Models of recruitment and rate coding organization in motor unit pools," J. Neurophysiol., vol. 70, pp. 2470-2488, Dec. 1993.

[15] M.-O. Gewaltig and M. Diesmann, "NEST (Neural Simulation Tool)," Scholarpedia, vol. 2, no. 4, p. 1430, 2007.

[16] Y. Nakamura, K. Yamane, Y. Fujita, and I. Suzuki, "Somatosensory computation for man-machine Interface from motion-capture data and musculoskeletal human model," IEEE Trans. Robot., vol. 21, no. 1, pp. 58-66, Feb. 2005.

[17] M. Djurfeldt et al., "Run-time interoperability between neuronal network simulators based on the MUSIC framework," Neuroinformatics, vol. 8, no. 1, pp. 43-60, Mar. 2010.

[18] F. Buchthal and H. Schmalbruch, "Motor unit of mammalian muscle," Physiol. Rev., vol. 60, no. 1, pp. 91-131, 1980.

[19] R. W. Banks, "An allometric analysis of the number of muscle spindles in mammalian skeletal muscles," J. Anat., vol. 208, no. 6, pp. 753-768, Jun. 2006.

[20] A. J. McComas, V. Galea, and H. de Bruin, "Motor unit populations in healthy and diseased muscles," Phys. Ther., vol. 73, pp. 868-877, 1993.

[21] A. Morrison, S. Straube, H. Plesser, and M. Diesmann, "Exact subthreshold integration with continuous spike times in discrete-time neural network simulations," Neural Comput., vol. 19, no. 1, pp. 47-79, Jan. 2007.

[22] E. Kandel, J. Schwartz, and T. Jessell, Principles of Neural Science, 4th ed. New York: McGraw-Hill, 2000.

[23] J. W. Fleshman, I. Segev, and R. E. Burke, "Electrotonic architecture of type-identified alpha-motoneurons in the cat spinal cord," J. Neurophysiol., vol. 60, no. 1, pp. 60-85, 1988.

[24] M. P. Mileusnic, I. E. Brown, N. Lan, and G. E. Loeb, "Mathematical models of proprioceptors: 1 . Control and transduction in the muscle spindle,” J. Neurophysiol., vol. 96, pp. 1772-1788, 2006.

[25] A. Prochazka and M. Grassini, "Models of ensemble firing of muscle spindle afferents recorded during normal locomotion in cats," $J$. Physiol., vol. 507, pp. 277-291, Feb. 1998.

[26] N. A. Al-Falahe, M. Nagaoka, and A. B. Vallbo, "Response profiles of human muscle afferents during active finger movements," Brain, vol. 113 , no. 2, pp. 325-344, 1990.

[27] S. Cullheim and J. O. Kellerth, "A morphological study of axons and recurrent axon collaterals of cat alpha-motoneurones supplying different functional types of muscle unit," J. Physiol., vol. 281, no. 1, pp. 301-313, Aug. 1978.

[28] R. E. Burke, "On the central nervous system control of Fast and Slow twitch motor units," in New Developments in Electromyography and Clinical Neurophysiology, J. E. Desmedt, Ed. Basel: Karger, 1973, pp. 69-94.

[29] E. Fournier, S. Meunier, E. Pierrot-Deseilligny, and M. Shindo, "Evidence for interneuronally mediated Ia excitatory effects to human quadriceps motoneurones," J. Physiol., vol. 377, no. 1, pp. 143-169, Aug. 1986.
[30] T. S. Buchanan, D. G. Lloyd, K. Manal, and T. F. Besier, "Neuromusculoskeletal modeling: Estimation of muscle forces and joint moments and movements from measurements of neural command," $J$. Appl. Biomech., vol. 20, no. 4, pp. 367-395, 2004.

[31] A. J. Fuglevand, "Mechanical properties and neural control of human hand motor units," J. Physiol., vol. 589, no. 23, pp. 5595-5602, 2011.

[32] A. F. Thilmann, S. J. Fellows, and E. Garms, "The mechanism of spastic muscle hypertonus," Brain, vol. 114, pp. 233-244, 1991.

[33] K. Ayusawa and Y. Nakamura, "Fast inverse dynamics algorithm with decomposed computation of gradient for wire-driven multi-body systems and its application to estimation of human muscle tensions," in 2nd IFToMM Int. Symp. Robot. Mechatron., 2011.

[34] M. J. D. Powell, The BOBYQA algorithm for bound constrained optimization without derivatives, Tech. Rep. NA2009/06, 2009,

[35] S. G. Johnson, The NLopt nonlinear-optimization package [Online]. Available: http://ab-initio.mit.edu/nlopt

[36] M. Sartori, M. Reggiani, E. Pagello, and D. G. Lloyd, "Modeling the human knee for assistive technologies," IEEE Trans. Biomed. Eng., vol. 59, no. 9, pp. 2642-2649, Sep. 2012.

[37] D. Farina, A. Holobar, R. Merletti, and R. M. Enoka, "Decoding the neural drive to muscles from the surface electromyogram," Clin. Neurophysiol., vol. 121, no. 10, pp. 1616-1623, Oct. 2010.

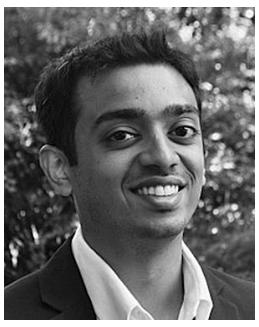

Manish Sreenivasa (M'15) received the M.Sc degree in mechanical engineering from Universität Duisburg-Essen, Germany, and the Ph.D. degree in systems engineering (humanoid robotics) from LAAS-CNRS, Toulouse, France. His doctoral work was focused on the development of human movement models to improve humanoid robot motion.

In the years since then, his research focus has been on the study of the neural control of movement, using interdisciplinary perspectives from neuroscience, biomechanics and robotics. Since 2013, he has also been working in close collaboration with the Orthopedic Department of Heidelberg University Hospital on developing computational methods for patient-specific rehabilitation.

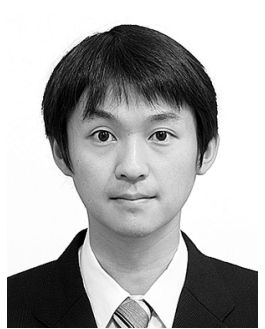

Ko Ayusawa received the B.S. degree in mechanical engineering, and the M.S. and Ph.D. degrees in mechano-informatics from the University of Tokyo, Japan, in 2006, 2008, and 2011, respectively.

He worked with the Department of Mechano-Informatics at the University of Tokyo, as a postdoctoral researcher from 2011 to 2012, and as a Project Assistant Professor in 2013. He is currently a researcher of the Intelligent Systems Research Institute, National Institute of Advanced Industria Science and Technology (AIST), Tsukuba. His research interests include identification of human/humanoid dynamics, motion control for humanoid robots, and kinematics and dynamics simulation for human musculoskeletal models.

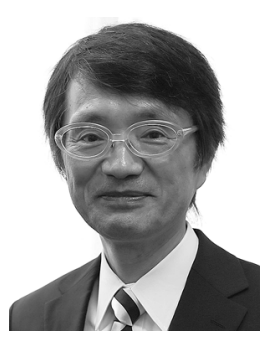

Yoshihiko Nakamura (F'11) received the Doctor of Engineering Degree from Kyoto University, in 1985.

He was Assistant Professor of Kyoto University, from 1982 to 1987, and then Assistant and Associate Professor of University of California, Santa Barbara, from 1987 to 1991 . Since 1991, he has been with University of Tokyo, Japan, and is currently Professor at Department of Mechano-Informatics. Humanoid robotics, cognitive robotics, neuro musculoskeletal human modeling, biomedical systems, and their computational algorithms are his current

fields of research.

Dr. Nakamura served as President of IFToMM (2011-2015). He is co-chairing IEEE-RAS Technical Committee on Robotics and Automation in Nuclear Facilities. He is Fellow of Japan Society of Mechanical Engineers, Fellow of Robotics Society of Japan, and Fellow of World Academy of Arts and Science. He is Foreign Member of Academy of Engineering Science of Serbia, and TUM Distinguished Affiliated Professor of Technische Universität München. 